\title{
Correspondence
}

\section{Gain-of-function experiments on $\mathrm{H} 7 \mathrm{~N} 9$}

Since the end of March 2013, avian influenza $A$ viruses of the H7N9 subtype have caused more than 130 human cases of infection in China, many of which were severe, resulting in 43 fatalities. Although this $\mathrm{A}(\mathrm{H} 7 \mathrm{~N} 9)$ outbreak is now under control, the virus (or one with similar properties) could re-emerge as winter approaches.

To better assess the pandemic threat posed by $\mathrm{A}(\mathrm{H} 7 \mathrm{~N} 9)$ viruses, investigators from the NIAID Centers of Excellence in Influenza Research and Surveillance and other expert laboratories in China and elsewhere have characterized the wild-type avian $\mathrm{A}(\mathrm{H} 7 \mathrm{~N} 9)$ viruses in terms of host range, virulence and transmission, and are evaluating the effectiveness of antiviral drugs and vaccine candidates. However, to fully assess the potential risk associated with these novel viruses, there is a need for further research, including experiments that may be classified as 'gain of function' (GOF).

Here we outline the aspects of the current situation that most urgently require additional research, our proposed studies, and risk-mitigation strategies.

The $\mathrm{A}(\mathrm{H} 7 \mathrm{~N} 9)$ virus haemagglutinin protein has several motifs that are characteristic of mammalianadapted and human influenza viruses, including mutations that confer human-type receptor binding and enhanced virus replication in mammals. The pandemic risk rises exponentially should these viruses acquire the ability to transmit readily among humans.

Reports indicate that several A(H7N9) viruses from patients who were undergoing antiviral treatment acquired resistance to the primary medical countermeasure neuraminidase inhibitors (such as oseltamivir, peramivir and zanamivir). Acquisition of

H7N 9 INFLUENZA RESEARCH

\section{Proposed gain-of-function experiments}

- Immunogenicity. To develop more effective vaccines and determine whether genetic changes that confer altered virulence, host range or transmissibility also change antigenicity.

- Adaptation. To assist with risk assessment of the pandemic potential of field strains and evaluate the potential of $A(H 7 N 9)$ viruses to become better adapted to mammals, including determining the ability of these viruses to reassort with other circulating influenza strains.

- Drug resistance. To assess the potential for drug resistance to emerge in circulating viruses, evaluate the genetic stability of mutations conferring drug resistance, and evaluate the efficacy of combination therapy with

resistance to these inhibitors by $\mathrm{A}$ (H7N9) viruses could increase the risk of serious outcomes of A(H7N9) virus infections.

The haemagglutinin proteins of $\mathrm{A}(\mathrm{H} 7 \mathrm{~N} 9)$ viruses have a cleavage site that is consistent with a low-pathogenic phenotype in birds. In the past, highly pathogenic $\mathrm{H} 7$ variants (with basic amino-acid insertions at the cleavage site that enable the spread of the virus to internal organs) have emerged from populations of low-pathogenic strains circulating in domestic gallinaceous poultry.

Normally, epidemiological studies and characterization of viruses from field isolates are used to inform policy decisions regarding public-health responses to a potential pandemic. However, classical epidemiological tracking does not give public-health authorities the time they need to mount an effective response to mitigate the effects of a pandemic antiviral therapeutics. Also, to determine whether $A(\mathrm{H} 7 \mathrm{~N} 9)$ viruses could become resistant to available antiviral drugs, and to identify potential resistance mutations that should be monitored during antiviral treatment.

- Transmission. To assess the pandemic potential of circulating strains and perform transmission studies to identify mutations and gene combinations that confer enhanced transmissibility in mammalian models (such as ferrets and guinea pigs). - Pathogenicity. To aid risk assessment and identify mechanisms, including reassortment and changes to the haemagglutinin cleavage site, that would enable circulating $A(H 7 N 9)$ viruses to become more pathogenic.

virus. To provide information that can assist surveillance activities — thus enabling appropriate public-health preparations to be initiated before a pandemic experiments that may result in GOF are critical.

Therefore, after review and approval, we propose to perform experiments that may result in GOF (see 'Proposed gain-offunction experiments').

All experiments proposed by influenza investigators are subject to review by institutional biosafety committees. The committees include experts in the fields of infectious disease, immunology, biosafety, molecular biology and public health; also, members of the public represent views from outside the research community. Risk-mitigation plans for working with potentially dangerous influenza viruses, including the 1918 virus and highly pathogenic avian $\mathrm{H} 5 \mathrm{~N} 1$ viruses, will be applied to conduct
GOF experiments with A(H7N9) viruses (see Supplementary Information at go.nature.com/ fstdy1). Additional reviews may be required by the funding agencies for proposed studies of $\mathrm{A}(\mathrm{H} 7 \mathrm{~N} 9)$ viruses.

The recent $\mathrm{H} 5 \mathrm{~N} 1$ virustransmission controversy focused on the balance of risks and benefits of conducting research that proved the ability of the $\mathrm{H} 5 \mathrm{~N} 1$ virus to become transmissible in mammals (see www.nature.com/mutantflu). These findings demonstrated the pandemic potential of $\mathrm{H} 5 \mathrm{~N} 1$ viruses and reinforced the need for continued optimization of pandemic-preparedness measures. Key mutations associated with adaptation to mammals, included in an annotated inventory for mutations in $\mathrm{H} 5 \mathrm{~N} 1$ viruses developed by the US Centers for Disease Control and Prevention, were identified in human isolates of A(H7N9) viruses. Scientific evidence of the pandemic threat posed by A(H7N9) viruses, based on $\mathrm{H} 5 \mathrm{~N} 1 \mathrm{GOF}$ studies, factored in risk assessments by public-health officials in China, the United States and other countries.

Since the $\mathrm{H} 5$ transmission papers were published, follow-up scientific studies have contributed to our understanding of host adaptation by influenza viruses, the development of vaccines and therapeutics, and improved surveillance.

Finally, a benefit of the $\mathrm{H} 5 \mathrm{~N} 1$ controversy has been the increased dialogue regarding laboratory biosafety and dualuse research. The World Health Organization issued laboratory biosafety guidelines for conducting research on $\mathrm{H} 5 \mathrm{~N} 1$ transmission and, in the United States, additional oversight policies and risk-mitigation practices have been put in place or proposed. Some journals now encourage authors to include biosafety and biosecurity descriptions in their papers, thereby raising the awareness of 
researchers intending to replicate experiments.

The risk of a pandemic caused by an avian influenza virus exists in nature. As members of the influenza research community, we believe that the avian A(H7N9) virus outbreak requires focused fundamental and applied research conducted by responsible investigators with appropriate facilities and risk-mitigation plans in place. To answer key questions important to public health, research that may result in GOF is necessary and should be done.

Ron A. M. Fouchier ${ }^{\star}$ Erasmus Medical Center, Rotterdam, the Netherlands.

r.fouchier@erasmusmc.nl

Yoshihiro Kawaoka* University of Wisconsin-Madison,

Wisconsin, USA.

kawaokay@svm.vetmed.wisc.edu ${ }^{*}$ On behalf of 22 co-authors (see go.nature.com/fstdy1 for full list).

\section{Extra oversight for H7N9 experiments}

The US Department of Health and Human Services (HHS) announces a new review process for certain gain-of-function (GOF) experiments on the avian influenza A (H7N9) virus, some of which are proposed this week by influenza scientists (R. A. M. Fouchier et al. Nature 500, 150-151; 2013).

Specifically, before being undertaken using HHS funds, any experiments that are reasonably anticipated to generate $\mathrm{H} 7 \mathrm{~N} 9$ viruses with increased transmissibility between mammals by respiratory droplets will undergo an additional level of review by the HHS.

The HHS review will consider the acceptability of these experiments in light of potential scientific and public-health benefits as well as biosafety and biosecurity risks, and will identify any additional risk-mitigation measures needed. The review will be carried out by a standing multidisciplinary panel of federal experts with backgrounds in public health, medicine, security, science policy, global health, risk assessment, US law and ethics.

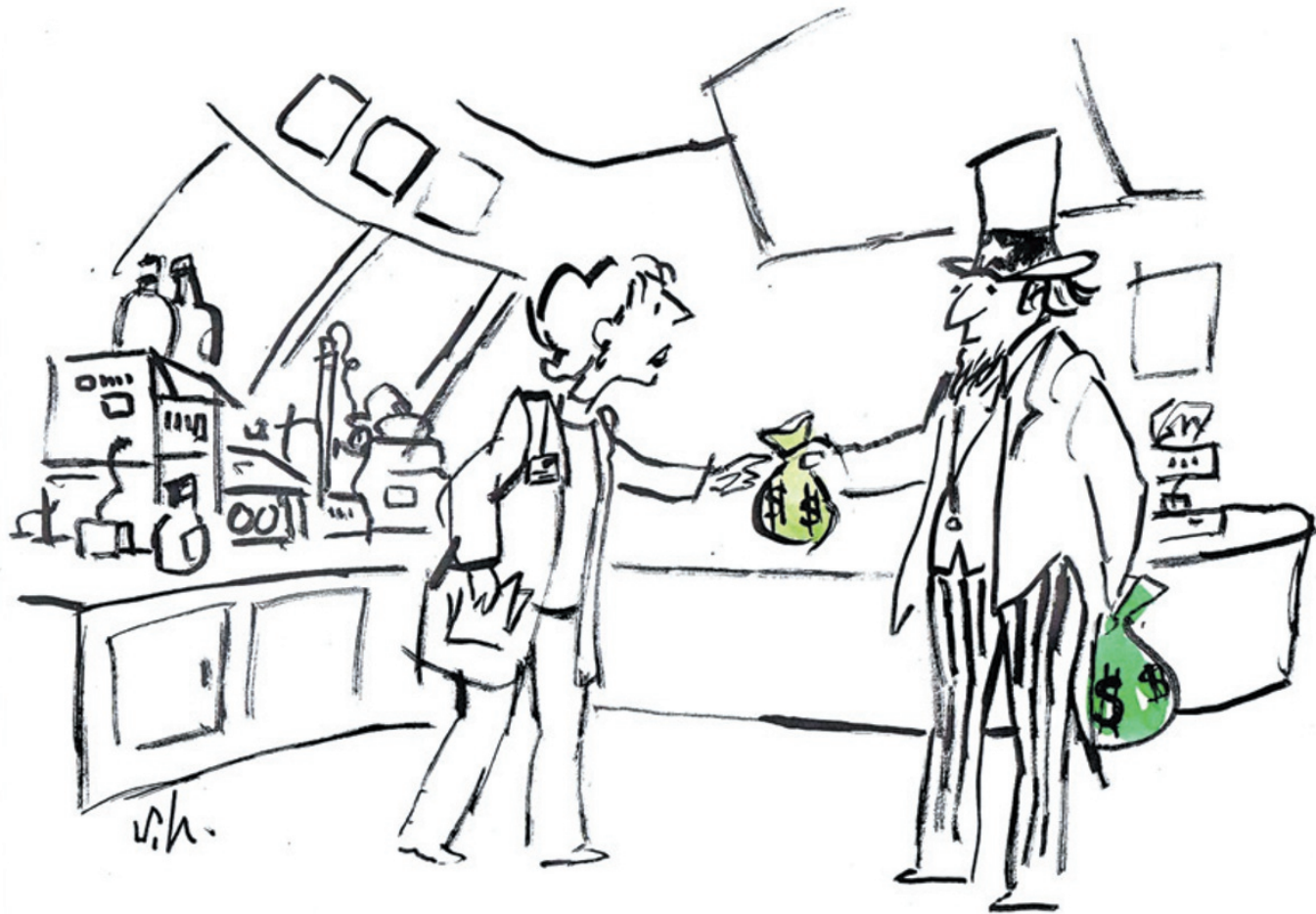

This approach, similar to that for certain $\mathrm{H} 5 \mathrm{~N} 1$ influenza virus experiments (see go.nature. com/vpmplf), allows the HHS to focus special oversight efforts on experiments of concern while allowing routine characterization and other fundamental research to proceed rapidly, thereby enabling a robust public-health response.

GOF studies can provide important insights into how the $\mathrm{A}(\mathrm{H} 7 \mathrm{~N} 9)$ virus adapts to mammalian hosts, causes disease and spreads to other hosts, but they may also pose biosafety and biosecurity risks. To ensure that research involving H7N9 virus is conducted safely and securely, the US Centers for Disease Control and Prevention recently re-examined the requisite biosafety conditions for conducting experiments involving H7N9 and, in June 2013, issued interim riskassessment and biosafety-level recommendations (see go.nature. com/gknn9a).

Harold W. Jaffe Centers for Disease Control and Prevention, Atlanta, Georgia, USA. Amy P. Patterson National Institutes of Health, Bethesda, Maryland, USA. pattersa@od.nih.gov Nicole Lurie Department of Health and Human Services, Washington DC, USA.

\section{Follow Obama's lead and take a pay cut}

In considering the impact of the US budget sequester on science (see, for example, Nature 499, 147-148; 2013), I see no mention of salary reductions. A 5\% reduction in the salaries of federally supported science staff, including administrative and agency personnel, would significantly reduce the need to cut science programmes.

US scientists are not paupers: NASA scientists, for instance, are paid up to US $\$ 160,000$ a year, with generous fringe benefits. Plenty of professors at leading US universities make much more. A 5\% cut to 12 -month and summer salaries would not leave anyone destitute.

Such a reduction would be much more effective than any presentations to Congress in showing that scientists care about their projects and are willing to share the pain of bringing US federal expenditure under control.

I doubt that any scientist would refuse to accept a grant offered on the proviso that the salary rate be reduced by $5 \%$, if the alternative were no grant at all. President Barack Obama took a pay cut to show the way.
Let's follow his lead. Peter Foukal Nahant, Massachusetts, USA. pvfoukal@comcast.net

\section{Three reasons for eco-label failure}

The fisheries industry promotes third-party eco-labels that signify sustainability, similar to those used in forestry and tourism (see Nature http://doi. org/nb5; 2013). In my view, these fail for three reasons.

First, consumers care strongly that labels for health and quality standards are accurate because they affect individuals, but care much less about eco-labels because their effects are spread across society.

Second, industries tend to use weak eco-labels in political games to avoid strong regulation.

Third, ineffective eco-labels closely mimic accurate ones. Because there are no adverse consequences for consumers who cannot tell them apart, a high proportion of mimics persists.

Eco-labels are thus no substitute for eco-laws. Ralf Buckley Griffith University, Gold Coast, Queensland, Australia. r.buckley@griffith.edu.au 